\title{
A New Weighted Region-based Hough Transform Algorithm for Robust Line Detection in Poor Quality Images of 2D Lattices of Rectangular Objects
}

\author{
Theocharis Tsenoglou ${ }^{*}$, Nikolaos Vassilas ${ }^{*}$ and Djamchid Ghazanfarpour ${ }^{+}$ \\ * Department of Informatics, Technological Educational Institute of Athens, Athens, Greece \\ + Département Mathématiques Informatique, XLIM (UMR CNRS 7252), Université de Limoges, Limoges, France
}

Received 23rd Oct. 2012; accepted 27th Feb. 2013

\begin{abstract}
In this work we present a novel kernel-based Hough Transform method for robust line detection in poor quality images of 2D lattices of rectangular objects. Following a preprocessing stage that specifies the connected regions of the image, the proposed method uses a kernel to specify each region's voting strength based on the following shape descriptors: a) its rectangularity, b) the orientation of the major side of its minimum area bounding rectangle (MBR), and c) the MBR's geometrical center. Experimental and theoretical analysis on the uncertainties associated with the geometrical center as well as the polar parameters of the MBR's major axis line equation allows for automatic selection of the parameters used to specify the shape of the kernel's footstep on the accumulator array. Comparisons performed on images of building facades taken under impaired visual conditions or with low accuracy sensors (e.g. thermal images) between the proposed method and other Hough Transform algorithms, show an improved accuracy of our method in detecting lines and/or linear formations. Finally, the robustness of the proposed method is shown in two other application domains those of, façade image rectification and skew detection and correction in rotated scanned documents.
\end{abstract}

Key Words: Pattern Recognition, Hough Transform, Rectangularity Measure, Building Facades, Night Images, Image Rectification, Skew Correction.

\section{Introduction}

Recently, there has been a significant interest within the computer vision, computer graphics, photogrammetry, artificial intelligence and geospatial database design communities in a variety of applications involving segmentation, classification, recognition and modeling of buildings and building facades [1-4], 3D reconstruction [5], generation of image mosaics [6], image patching (e.g. for generating panoramas or street views) [7,8], image matching (e.g. finding correspondences in images of building facades) [9], content-based image retrieval from building databases [10,11] and automatic generation of large photorealistic 3D city models [12].

However, most of the above techniques assume that the images have been obtained under daylight vision conditions. None that we are aware of has taken into account night view images of buildings possibly of low resolution and obtained under bad weather conditions (an excellent thesis on modeling weather phenomena

Correspondence to: theoharis@hotmail.com

Recommended for acceptance by $<$ Georgios Triantafyllidis $>$

ELCVIA ISSN: 1577-5097

Published by Computer Vision Center / Universitat Autonoma de Barcelona, Barcelona, Spain 
and recovering pertinent scene properties from one or more images taken under poor weather conditions is presented in [13]). Under such circumstances, the typical preprocessing step of line or segment extraction using variants of the Hough Transform (HT) [14,15] for vanishing point estimation $[16,17]$ and image rectification [18] is severely affected by the poor quality of edges detected in the image.

Moreover, although edge detection usually precedes application of the HT, in order to reduce the number of foreground pixels to those "hopefully" relevant and avoid the unnecessary computations otherwise carried out on a binary (thresholded or segmented) image, it is often the case that the image content is better described with the solid regions of the objects rather than their boundaries. This is the case when the images are blurred (e.g. due to fog, mist, out-of-focus), visually impaired (e.g. night views), noisy (e.g. snow, rain, dust, flying debris) or obtained with poor quality and/or low resolution sensors (e.g. thermal IR imagery). In addition, there are cases, such as when we are interested in finding prevalent line orientations that may be different from the regions' edge orientations or when we seek implicit axes passing through the central part of - possibly aligned - regions, rendering such a preprocessing undesirable.

When we seek to find linear structural formations in night images of office buildings with bright pixels corresponding to lit windows, it is natural to consider HT variants that take into account shape, orientation and alignment of the bright image regions. Such a general purpose region-based HT variant, designed to detect straight lines, linear formations and dominant orientations in black \& white images has been proposed in [19]. The method was shown to exhibit high accuracy in determining linear formations as well as prevalent orientation in a number of real and synthetic images [20,21] and compared favorably to traditional edge-based HT methods. The shape descriptors obtained by the abovementioned variant assume that the regions are fitted by ellipses. However, it appears that in the context of night view images of buildings and building facades, the regions are best approximated by rectangles rather than ellipses, since they mostly correspond to lit windows and other rectangular architectural structures. In addition, since, usually, in office buildings there is a spatial arrangement of windows and doors in regular grids, the HT variant proposed in this work should also be appropriate for finding linear formations and prevalent line orientations.

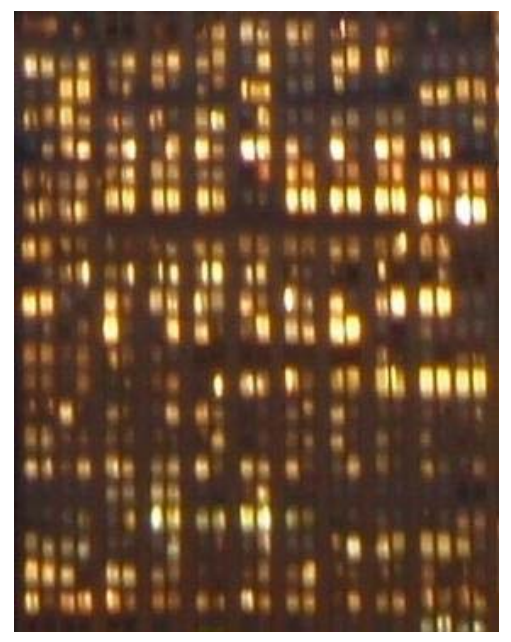

(a)

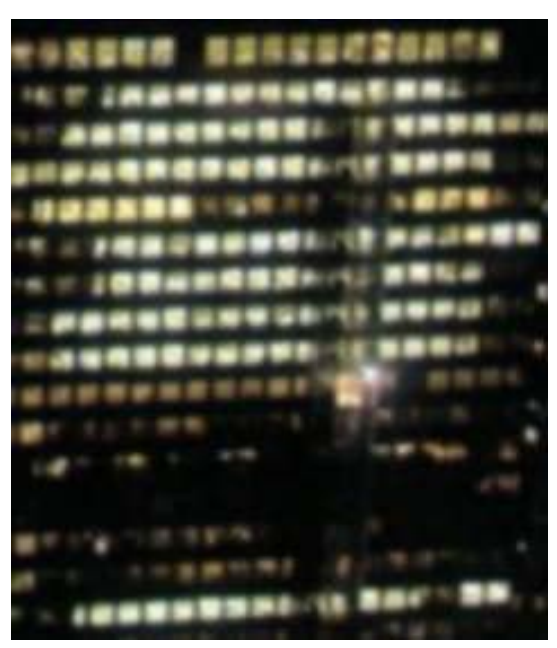

(b)

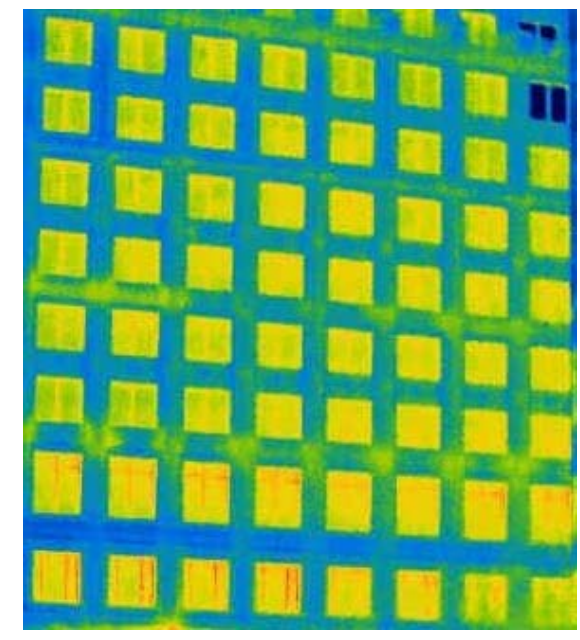

(c)

Fig. 1 Noisy night view images (the rightmost image is with a thermal IR sensor) of office buildings with quite low resolutions: a) $286 \times 367$ pixels, b) $238 \times 275$ pixels and c) $292 \times 328$ pixels

The method presented in this work is appropriate for robust detection of implicit lines - passing through the regions' geometrical centers - as well as of linear formations in noisy lattices of rectangular objects such as poor quality night view images of buildings with repetitive but incomplete structural information. Fig. 1 shows the type of images used in this work: noisy and low resolution night images of office buildings with some lit windows. To this end, we first binarize the image using Otsu's method [22] for automatic thresholding of the original gray level histogram, then we decompose the binary image into connected regions and compute a rectangularity score for each region based on the Minimum Bounding Rectangle (MBR) method [23], next we filter out regions with low rectangularity scores and, finally, we introduce a 
product of Gaussians kernel that is used to specify the region's contribution to the accumulator array and which models uncertainties associated with the MBR's geometrical center and major side orientation. The proposed technique has several strong points:

- It works well in images where edge detectors cannot find reliable edges, such as night building views, even under bad weather conditions (e.g. mist, fog, rain, snow, storm), and where only some windows are lit.

- It can estimate with high accuracy prevalent line orientations.

- It has improved line localization accuracy over other region-based HT methods.

- It can be used, in conjunction with the RANSAC technique [24] and appropriate clustering, to specify homographies in 3D image rectification applications.

- It is appropriate in cases where the required lines are axes located in the interior of objects.

- It filters out lines not produced by the rectangular regions in the image.

Finally, the validity of the proposed method is also shown in line extraction from low accuracy thermal IR images as well as in a completely different problem: it is used for skew detection and correction in scanned documents which is a typical preprocessing step in document image analysis applications. Extraction of rectangular blocks from the document images is performed using the run length smearing algorithm (RLSA).

Section 2 presents the preprocessing steps applied to a typical image as well as the experimental computation of the uncertainties associated with region orientation, Section 3 presents the proposed regionbased HT algorithm, Section 4 presents experiments and comparisons with other HT algorithms and, finally, Section 5 presents the conclusions of this work.

\section{Preprocessing and Experimental Computation of Orientation Uncertainties}

In this section we present the preprocessing steps followed throughout all experiments of this work as well as the experimental estimation of the uncertainty associated with the orientation measurement of the minimum bounding rectangle of a region.

\subsection{Preprocessing}

The preprocessing steps that are applied to an input image before extracting lines, detecting linear formations or specifying prevalent orientations are as follows:

- Color-to-grayscale image conversion. This step transforms input images to monochrome ones. In the case of RGB images, we compute the intensity I at some pixel by mixing the three color components according to $\mathrm{I}=0.299 \mathrm{R}+0.587 \mathrm{G}+0.114 \mathrm{~B}$ and quantizing to the given grayscale.

- Binarization. We obtain a black \& white image by thresholding the grayscale image using Otsu's automatic threshold selection method. This technique works well with the bimodal histograms of bright objects in night images. However, when the image histograms are more complicated other binarization techniques such as adaptive thresholding or even involving sophisticated region segmentation should be considered.

- Morphological operations. Mathematical morphology with 3x3 structuring elements is used in order to separate touching objects and remove slender protrusions, intrusions and small holes. This step is necessary to improve fidelity at the stage of rectangularity assessment of each region. 
- Extraction of connected components and labeling. In this stage the binary image is decomposed into connected regions using the 4-neighbors labeling algorithm.

- Area computation and filtering. To improve the significance of the overall methodology, we found it necessary to compute the area of each region and then filter out those regions whose area is below some prespecified threshold. In fact, due to noise and discretization effects, a small region is more prone to measurement errors than a larger ones when assessing its rectangularity as well as the geometrical center and principal axis orientation of its minimum bounding rectangle. In all experiments, we selected to remove all regions of 15 pixels or less.

- Specification of minimum bounding rectangles. To each of the remaining regions we fit the minimum bounding rectangle (MBR) using d'Errico's matlab code [25]. This algorithm improves computational speed by rotating the region only in the directions of the convex hull (polygon) sides and finding the corresponding bounding box for each such rotation angle. The MBR will then be the so found minimum area bounding box.

- Computation of rectangularity scores. Having computed the MBR, we assess the rectangularity score of each region using the following rectangularity measure $[23,26]$ :

$$
\mathrm{R}=\mathrm{A}_{0} / \mathrm{A}_{\mathrm{MBR}}
$$

where $A_{0}$ is the region's area and $A_{M B R}$ is the area of the MBR.

- Rectangularity filtering. The rectangularity scores obtained using the above measure are in $[0,1]$, with circular objects having $\mathrm{R}=\pi / 4$. Lower scores correspond to ragged regions or regions with irregular shapes. In all experiments we filter out regions having a rectangularity score below some user-defined threshold $\mathrm{R}_{\min }$ (set to 0.65 in the sequel), as they are considered noise or not belonging to the class of rectangular objects, prior to the application of the proposed kernel-based Hough transform algorithm.

\subsection{Experimental computation of the uncertainty of MBR's orientation}

In this section we present the experimental computation of the uncertainty $\sigma_{\theta^{\prime} \mid \varphi}$ associated with the estimate of the MBR's true orientation $\theta^{\prime}$ given the measured orientation $\varphi$. This uncertainty is related to the discretization effects that may lead to discrepancies between a rotated rectangle by some known angle and the estimate of that angle from the orientation of the MBR's major side. In addition, the uncertainty depends on the region's area and on the rectangularity score. In order to assess $\sigma_{\theta^{\prime} \varphi}$ we performed the following experiment.

Assume that we process a region with a rectangularity score $\mathrm{R}$ and with a true principal axis orientation of its MBR at $\theta^{\prime}$ degrees (see Fig. 2). Also, assume that the length of the MBR's major side is $\mathrm{N}$ and that the Hough space is discretized with an angular quantization step of $\Delta \theta$ (equal to $1^{\circ}$ in all experiments).

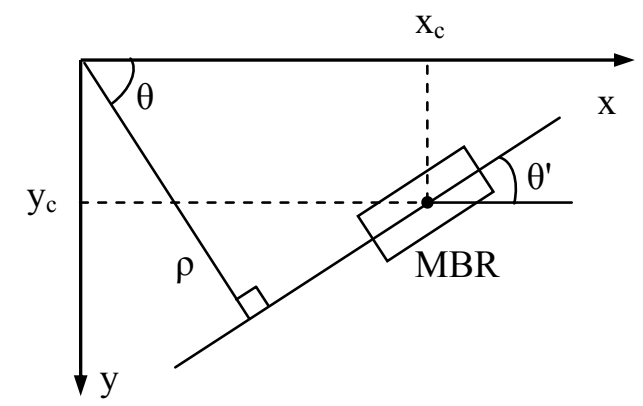

Fig. 2 The $\rho, \theta$ parameterization of a region's MBR principal axis. 
Let $\varphi$ be the measured (observed) MBR orientation and let $\theta^{\prime}$ be the (estimated) true rotation of the rectangular region. To simplify matters, we will assume that the rotation of an input image (along with all its rectangular regions) may take only the discrete values $\theta_{\mathrm{i}}{ }^{\prime}=\mathrm{i} \cdot \Delta \theta^{\prime}$ where $\mathrm{i} \in \mathrm{Z}$ and $\Delta \theta^{\prime}$ is the input image rotation step. Moreover, we will assume that $\Delta \theta^{\prime}=\Delta \theta$, i.e. $\theta_{i}{ }^{\prime}=i \cdot \Delta \theta$. In fact, this assumption will be satisfied in the controlled experiment with synthetic images of known true rotations described below.

Since, in general, $\varphi$ is different from the true rotation $\theta^{\prime}$, due to discretization effects, we want an estimate of $\theta^{\prime}$. In order to do this, we will consider $\theta^{\prime}$ as a discrete random variable with values $\theta_{i}^{\prime}$. We seek the conditional mean and variance of $\theta^{\prime}$ given observation $\varphi$, that is $\mu_{\theta^{\prime} \varphi}$ and $\sigma_{\theta^{\prime} \varphi}^{2}$ given by:

and

$$
\mu_{\theta^{\prime} \varphi}=\Sigma_{\mathrm{i}} \mathrm{P}\left(\theta^{\prime}=\theta_{\mathrm{i}}{ }^{\prime} \mid \varphi\right) \theta_{\mathrm{i}}{ }^{\prime}
$$

$$
\sigma_{\theta^{\prime \prime} \varphi}^{2}=\Sigma_{\mathrm{i}} \mathrm{P}\left(\theta^{\prime}=\theta_{\mathrm{i}}^{\prime} \mid \varphi\right) \theta_{\mathrm{i}}^{\prime 2}-\mu_{\theta^{\prime} \varphi}^{2}
$$

The posterior probabilities $\mathrm{P}\left(\theta^{\prime}=\theta_{i}^{\prime} \mid \varphi\right)$ are estimated experimentally from synthetic images of perfect rectangles with various sizes and at various distances from the origin of rotation (see Fig. 3). We implicitly assume that the dependence of $\sigma_{\theta^{\prime} \varphi}$ on the region's area is reflected to a dependence of $\sigma_{\theta^{\prime \prime} \varphi}$ on the length of its MBR's major side The so estimated $\sigma_{\theta^{\prime} \varphi}$ (conditional standard deviation) will represent the uncertainty on the true rotation angle and should depend on $\mathrm{R}, \mathrm{N}$ and $\varphi$.

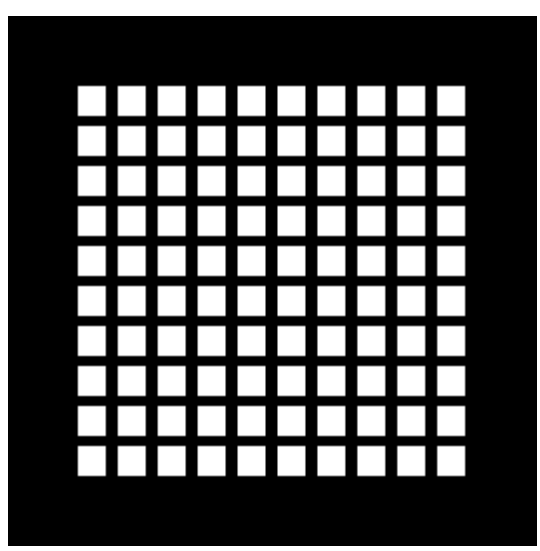

(a)

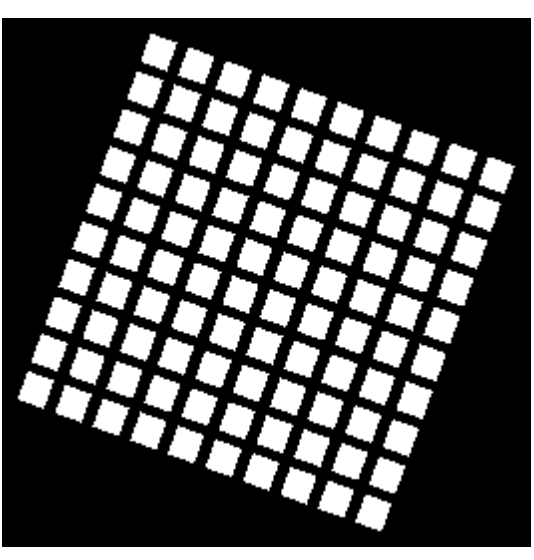

(b)

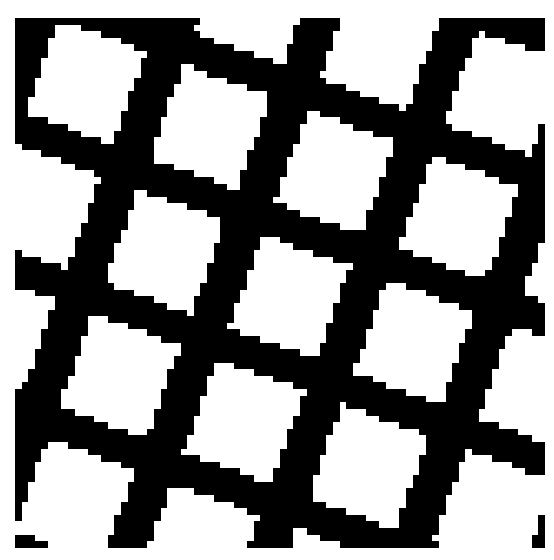

(c)

Fig. 3 a) One of the synthetic images showing a regular grid pattern of rectangles having a size of $15 \times 14$ pixels, b) the clockwise rotation of (a) by $20^{\circ}$ using the nearest neighbor interpolation method and c) a detail of the rotated rectangles showing different alterations due to the discretization effects.

The experimental setup and the corresponding steps followed for the uncertainty estimation of the true lattice rotation is presented below:

a) First, we generated 48 synthetic images of 100 rectangles each arranged in a 10x10 regular grid pattern (see Fig. 3) with dimensions of $\mathrm{N} x(\mathrm{~N}-1)$ pixels where $\mathrm{N}$ is the length of the major side and $(\mathrm{N}-1)$ the length of the minor side and $\mathrm{N}=3,4, \ldots, 50$. Experimentation on various sizes was necessary to assess the dependence of $\sigma_{\theta^{\prime} \mid \varphi}$ on the size of the rectangle since the larger the rectangle sides the more accurate the estimates of its orientation will be. Moreover, the spatial arrangement of the rectangles accounts for different placements in the image resulting to different discretization errors (see Fig. 3c).

b) Then, we rotated each synthetic image in increments of $\Delta \theta=1^{\circ}$ covering the range of $\left[-50^{\circ}, 50^{\circ}\right]$ using the nearest neighbor interpolation method. For each rotated image we computed the MBRs and for each such MBR we recorded its major axis orientation, i.e. its measured angle $\varphi$.

c) Next, for each $\mathrm{N}$, we estimated $\mathrm{P}\left(\theta^{\prime}=\theta_{\mathrm{i}^{\prime}} \mid \varphi\right)$ as follows: For each $\varphi$ we recorded all possible actual (true) rotation angles $\theta_{\mathrm{i}}^{\prime}$ that resulted to at least one rotated rectangular region with MBR orientation 
equal to $\varphi$. Let the set of these $\theta_{i}^{\prime}$ be $S(\varphi)$. In addition, for each $\theta_{i}^{\prime} \in S(\varphi)$ we recorded the number of rectangles $\mathrm{C}_{\varphi}\left(\theta_{\mathrm{i}}^{\prime}\right)$ whose MBR orientation was equal to $\varphi$. The a posteriori probabilities are then computed as:

$$
\mathrm{P}\left(\theta^{\prime}=\theta_{\mathrm{i}}^{\prime} \mid \varphi\right)=\mathrm{C}_{\varphi}\left(\theta_{\mathrm{i}^{\prime}}^{\prime}\right) / \Sigma_{\theta_{\mathrm{k}}^{\prime} \in \mathrm{S}(\varphi)} \mathrm{C}_{\varphi}\left(\theta_{\mathrm{k}}^{\prime}\right)
$$

By substituting (4) in (2) and (3), we obtain estimates of the uncertainties $\sigma_{\theta^{\prime} \varphi \varphi}$ for each $\mathrm{N}$ and $\varphi$ (for $\mathrm{N}>50$ we assume the same uncertainties as those for $\mathrm{N}=50$ ). Fig. 4a shows the dependence of $\sigma_{\theta^{\prime} \varphi}$ on $\mathrm{N}$ for $\varphi=-45^{\circ}$ and $\varphi=0^{0}$. From this diagram it is clear that the uncertainty tends to zero as the size grows larger. Fig. $4 \mathrm{~b}$ shows the dependence of $\sigma_{\theta^{\prime} \varphi}$ on $\varphi$ for $\mathrm{N}=10,15$ and 30 .
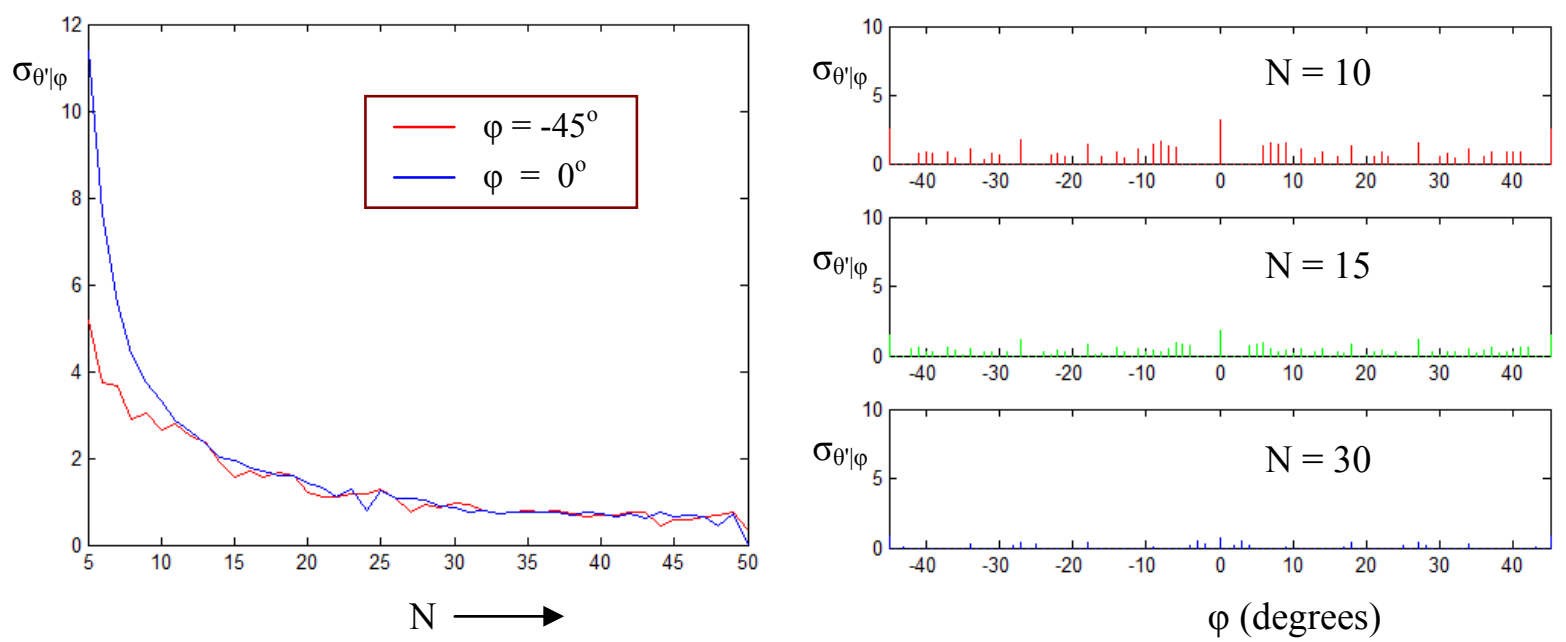

Fig. 4 a) Plots of uncertainties $\sigma_{\theta^{\prime} \varphi}$ with respect to $\mathrm{N}$ for two different rotation estimates and, b) plots of $\sigma_{\theta^{\prime} \varphi}$ with respect to $\varphi$ for three different major side lengths.

d) Finally, since the uncertainty is clearly affected by the region's rectangularity, $\sigma_{\theta^{\prime} \varphi}$ is taken to be exponentially related to the rectangularity score according to the following equation

$$
\sigma_{\theta^{\prime} \mid \varphi}(\mathrm{R})=\sigma_{\theta^{\prime} \mid \varphi} 2^{(1-R) /(1-R \min )}
$$

The above assumes that $R_{\min } \leq \mathrm{R} \leq 1$ and that at $\mathrm{R}_{\min }$ the uncertainty on $\theta^{\prime}$ is doubled. In the sequel we will drop the dependence on $R$ and refer to the above overall uncertainty of equation (5) as $\sigma_{\theta^{\prime} \varphi \varphi}$.

\section{The Proposed Region-based HT Method}

The original HT is a popular and powerful technique for the detection of straight lines in binary images. Given a binary image, we are interested in the detection of straight lines whose points $\left(\mathrm{x}_{\mathrm{c}}, \mathrm{y}_{\mathrm{c}}\right)$ are parameterized by

$$
\rho(\theta)=x_{c} \cos \theta+y_{c} \sin \theta
$$

where $\rho(\theta)$ is the distance of the origin from a particular line and $\theta$ is the angle formed by the normal to the line and the $\mathrm{x}$-axis (see Fig. 2).

In the original HT all foreground pixels contribute the same amount to those accumulator array cells that correspond to lines passing through them. Preferential weighting of certain pixels can be achieved by introducing a voting kernel [27]. However, since the original HT is usually applied to edge-images, we will also consider a weighted region-based HT variant [19] which uses a weighting function to specify the region's contribution to the accumulator array that depends on the following shape descriptors: a) region 
area, b) elongation (major to minor axis length ratio of the "best fitting ellipse"), c) orientation of the region's principal axis, and d) the region's geometrical center. It has been experimentally shown that this method is appropriate for finding lines and linear formations as well as prevalent orientations in a variety of applications [20,21]. This HT variant will be used for comparison purposes with our proposed method in several applications.

The HT variant that we propose in this work employs a novel suitably defined voting kernel to reduce interference effects and avoid spurious accumulator array maxima even in very noisy images. To allow for significant misalignments (e.g. due to noise and discretization) of the rectangular regions in the lattice, the method incorporates both tolerance on the MBR position as well as tolerance on its orientation.

Before proceeding, we will assume that the region orientation $\theta^{\prime}$ as well as its geometrical center $(\mathrm{x}, \mathrm{y})$ are independent random variables with mean and variance given by

$$
\mathrm{E}\{\mathrm{x}\}=\mathrm{x}_{\mathrm{c}}, \quad \mathrm{E}\{\mathrm{y}\}=\mathrm{y}_{\mathrm{c}}, \quad \sigma_{\mathrm{x}}^{2}=\sigma_{\mathrm{y}}^{2}=\sigma_{\mathrm{gc}}^{2}
$$

and

$$
\mathrm{E}\left\{\theta^{\prime} \mid \varphi\right\}=\varphi, \quad \sigma_{\theta^{\prime} \varphi}^{2} \text { : experimentally determined and passed through equation (5) }
$$

In fact, (7) and (8) assume that the expected values of the region's geometrical center and orientation i.e., $\left(\mathrm{x}_{\mathrm{c}}, \mathrm{y}_{\mathrm{c}}\right)$ and $\varphi$ respectively, are directly measured from the region's MBR while the uncertainty expressed as the standard deviation for these random variables is assumed equal to $\sigma_{\mathrm{gc}}$ regarding the location of the geometrical center and given by the experimentally determined $\sigma_{\theta^{\prime} \mid \varphi}$ regarding $\theta^{\prime}$.

From Fig. 2, and due to the simple first-order relation between the random variables $\theta$ and $\theta^{\prime}$, i.e. $\theta= \pm 90^{\circ}$ - $\theta^{\prime}$, it is easy to show that $\sigma_{\theta \mid \varphi}^{2}=\sigma_{\theta^{\prime} \varphi}^{2}$. Having the means and variances of $\mathrm{x}, \mathrm{y}$ and $\theta$ we are now able to estimate the uncertainty associated with $\rho$ and propose the new kernel. Actually from (6) and (7) we obtain:

$$
\mathrm{E}\{\rho \mid \theta\}=\mathrm{E}\{\mathrm{x} \cos \theta+\mathrm{y} \sin \theta \mid \theta\}=\mathrm{E}\{\mathrm{x}\} \cos \theta+\mathrm{E}\{\mathrm{y}\} \sin \theta=\mathrm{x}_{\mathrm{c}} \cos \theta+\mathrm{y}_{\mathrm{c}} \sin \theta=\rho(\theta)
$$

where $\rho(\theta)$ is the parametric form (see (6)) of the principal MBR axis passing through its geometrical center and

$$
\begin{aligned}
\sigma_{\rho \mid \theta}^{2} & =\mathrm{E}\left\{\rho^{2} \mid \theta\right\}-\mathrm{E}\{\rho \mid \theta\}^{2}=\mathrm{E}\left\{\mathrm{x}^{2}\right\} \cos ^{2} \theta+\mathrm{E}\left\{\mathrm{y}^{2}\right\} \sin ^{2} \theta+2 \mathrm{E}\{\mathrm{xy}\} \cos \theta \sin \theta \\
& -\mathrm{x}_{\mathrm{c}}{ }^{2} \cos ^{2} \theta-\mathrm{yc}_{\mathrm{c}}{ }^{2} \sin ^{2} \theta-2 \mathrm{x}_{\mathrm{c}} \mathrm{y}_{\mathrm{c}} \cos \theta \sin \theta=\sigma_{\mathrm{x}}{ }^{2} \cos ^{2} \theta+\sigma_{\mathrm{y}}{ }^{2} \sin ^{2} \theta=\sigma_{\mathrm{gc}}{ }^{2}
\end{aligned}
$$

Finally, the proposed kernel used to specify the way a particular region casts its votes will be given by:

where

$$
h(\rho, \theta, \mathrm{R}, \varphi)=f(\mathrm{R}, \theta, \varphi) \exp \left[-(\rho-\rho(\theta))^{2} / \sigma_{\mathrm{gc}}{ }^{2}\right]
$$

$$
f(\theta, \mathrm{R}, \varphi)=g(\mathrm{R}) \exp \left[-(\theta-\varphi)^{2} /(\sigma-\mathrm{R})^{2}\right]
$$

and

$$
g(\mathrm{R})=0.1+0.9 /(1+\exp (-\mathrm{w}))
$$

where $g(\cdot)$ is a logistic function of $\mathrm{R}$ used to regulate voting strength according to region's rectangularity score, $\mathrm{w}=10\left(\mathrm{R}-\mathrm{R}_{\min }\right) /\left(1-\mathrm{R}_{\min }\right)-4$ and $\mathrm{R}_{\min }$ is the minimum allowed rectangularity score before filtering out the region.

Although (14) has been shown adequate in finding linear formations in noisy night view images [27], by requiring that the regions should be perfectly aligned in order, for all of them, to contribute to the same accumulator cell (i.e, to the same line) we compromised the accuracy of line localization. In other words, using the kernel described in (14) and due to digitization effects that may cause misalignments, a line could be voted only by a few perfectly aligned geometrical centers although many more regions might actually participate in a particular linear formation. Therefore, it is evident that by introducing a tolerance on the actual location of the line will render the HT method more robust to small region misalignments and will improve line localization.

The proposed technique enhances (14) with a second Gaussian factor that accounts for lines having the same $\theta$ but different $\rho$. In particular, for each line $(\rho(\theta), \theta)$ passing from $\left(\mathrm{x}_{\mathfrak{c}}, \mathrm{y}_{\mathrm{c}}\right)$, where $\rho(\theta)$ is computed from 
(6), we also increment the accumulator cells for the zone $\{(\rho(\theta)-\delta, \theta), \ldots,(\rho(\theta)+\delta, \theta)\}$ of parallel lines where $\delta$ is the maximum deviation around $\rho(\theta)$, i.e. $|\rho-\rho(\theta)| \leq \delta$. Hence, the kernel's footstep in Hough space will have the shape of a sinusoidal ribbon. A value of 2 for the uncertainty $\sigma_{\rho \mid \theta}=\sigma_{\mathrm{gc}}$ and a value of 1.3rads for the tolerance $\sigma$ in (14) are found reasonable and used throughout the experiments.

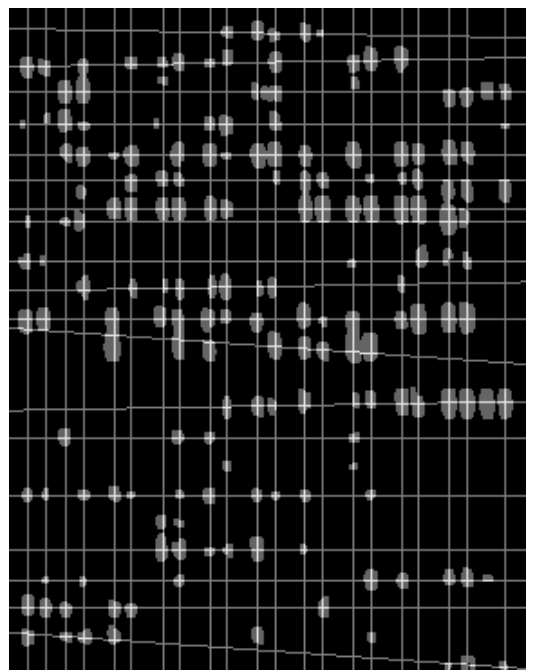

(a)

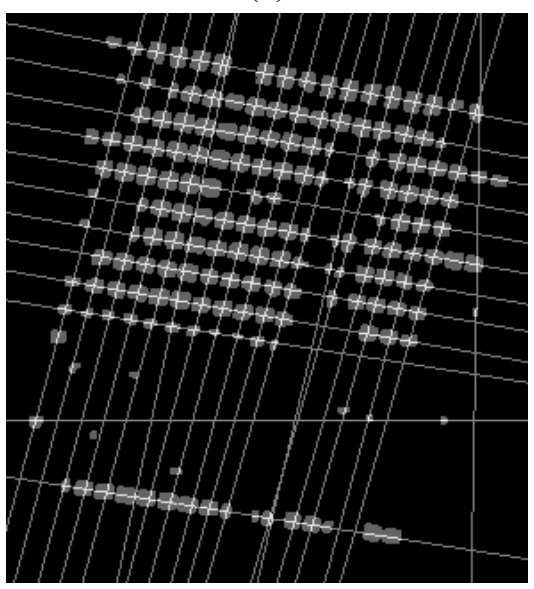

(d)

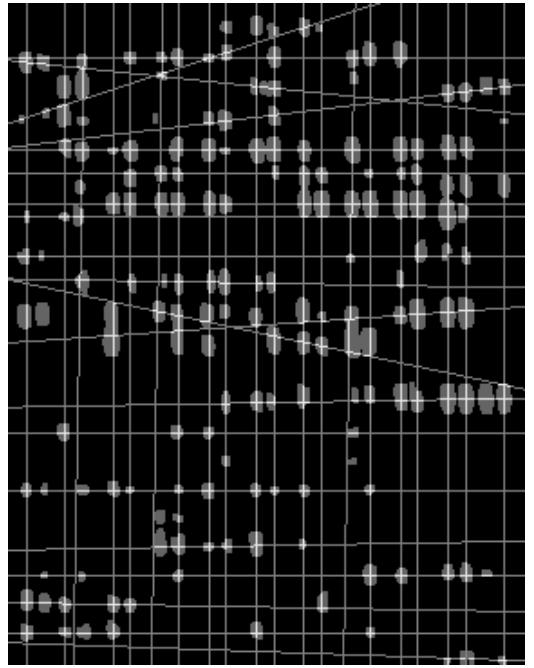

(b)

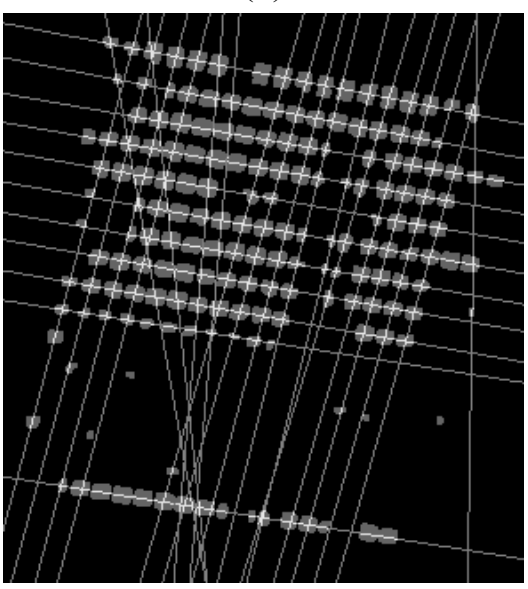

(e)

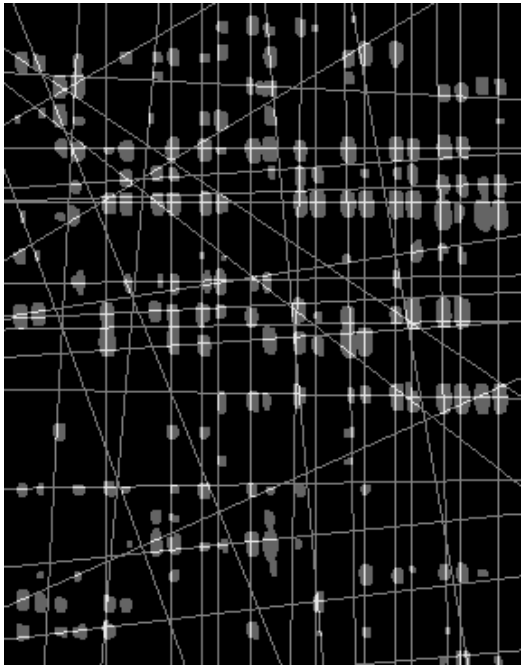

(c)

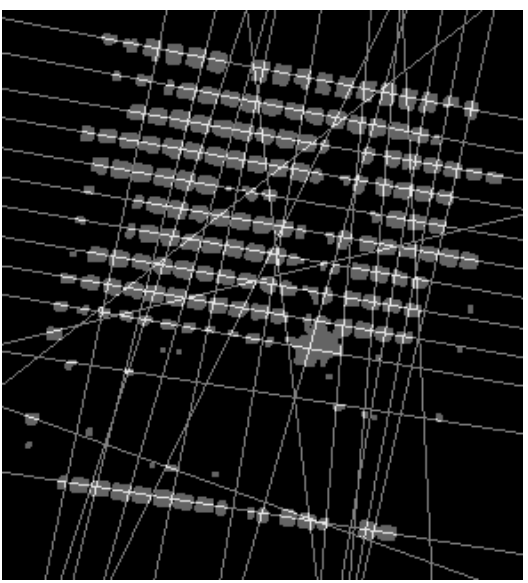

(f)

Fig. 5 (a), (b), (c) show the 40 most prominent lines extracted from Fig. 1a for the proposed, $f$-kernel and ellipse-based HT variants, respectively. Similarly, (d), (e), (f) show the 30 most prominent lines extracted from a rotated by $12^{\circ}$ image of Fig. $1 \mathrm{~b}$ for the three methods, respectively.

\section{Experimental Results}

Several experiments have been performed in order to assess the suitability of the proposed kernel-based HT in finding major lines and/or linear structures in lattices of rectangular objects such as noisy night view images of buildings and RLSA-preprocessed scanned documents for skew detection and correction.

The most prominent lines extracted with the proposed $h$-kernel of eq. (13) from Fig. 1a and which correspond to the 40 highest peaks of the accumulator array are shown in Fig 5a. Also shown in Figs. 5b and $5 \mathrm{c}$ are the corresponding 40 most prominent lines obtained with the $f$-kernel HT variant of eq. (14) and the ellipse-based HT method respectively using the same binarization, filtering and peak selection procedures as for the proposed method. Careful visual examination of these images shows that the rectangle-based methods have improved quality in finding linear formations compared to the ellipse-based method. In addition, the 
proposed method has higher line detection capabilities and improved line localization compared to the $f$ kernel variant.

Also shown in Fig. 5d are the 30 most prominent lines extracted with the proposed variant from Fig. $1 \mathrm{~b}$ after it has been rotated by $12^{\circ}$ in the clockwise direction. In these experiments the proposed method outperforms the other two HT variants regarding line detection rate and line localization accuracy. Moreover, the $f$-kernel variant shows superior performance compared to the ellipse-based HT variant.

Experiments run on a 4GB, Dual-CPU AMD Turion 64bit X2 TL-56 laptop, show that the overall execution times range from 0.4 to 1.2 secs for all algorithms. The experimental results show that the preprocessing outlined in Sec. 2.1 (common to both the proposed and f-kernel HT algorithms) is about 2.4 times more demanding than the corresponding preprocessing needed to specify the best fitting ellipses for the ellipse-based HT variant. For a typical example, such as a rotation of Fig. 1, the preprocessing times are 0.74 and 0.31 secs respectively. On the other hand, regarding the HT execution times, the f-kernel is the fastest algorithm, the proposed algorithm is the slowest (due to the uncertainty on the geometrical center of each region which increases computational load for the generation and decomposition of the accumulator array) and the ellipse-based variant is in-between. Typical times for the same rotated figure are: $0.08 \mathrm{sec}$ for the $\mathrm{f}$ kernel, $0.12 \mathrm{sec}$ for the ellipse-based and $0.21 \mathrm{sec}$ for the proposed algorithms.

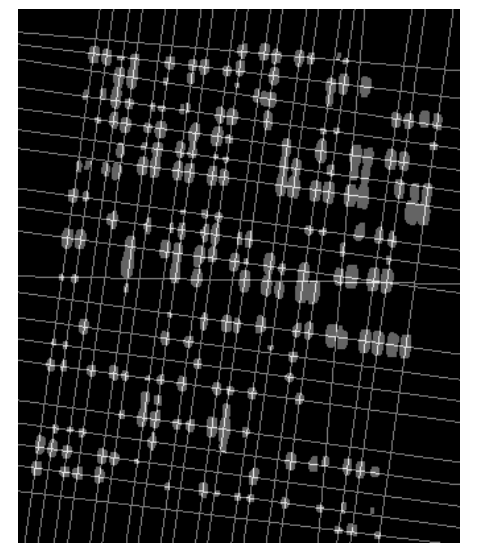

(a)

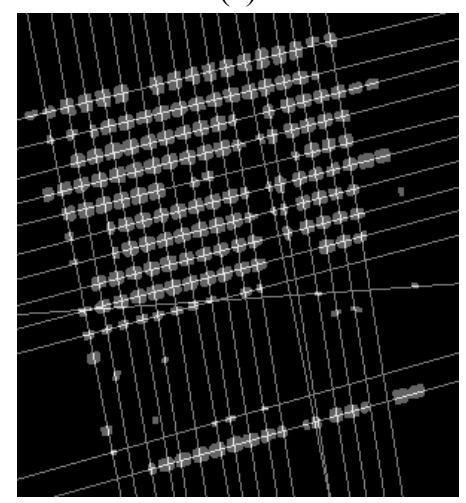

(d)

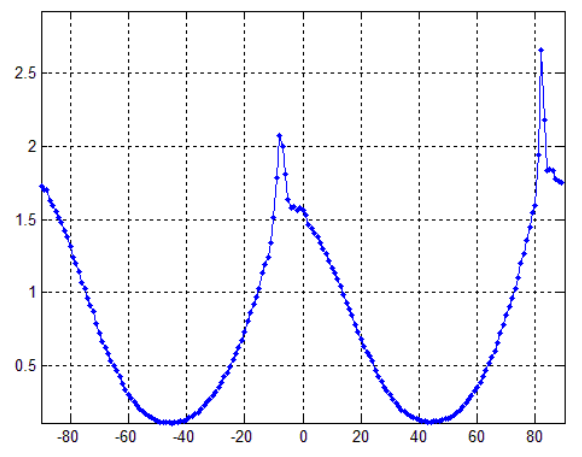

(b)

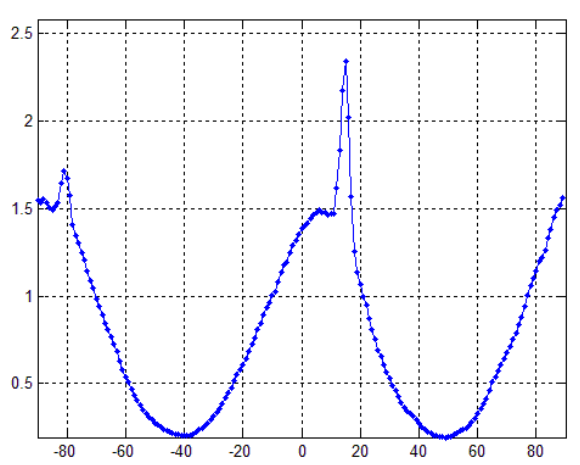

(e)

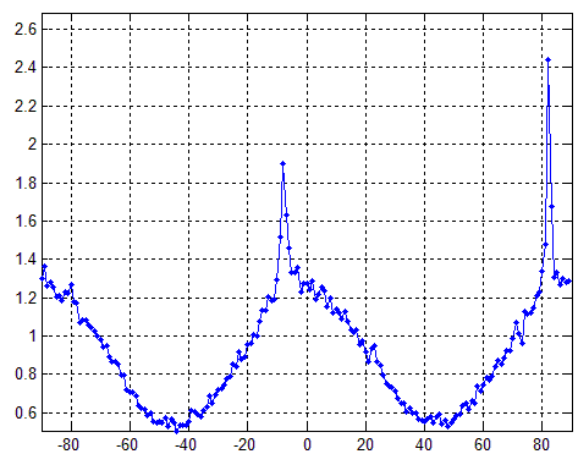

(c)

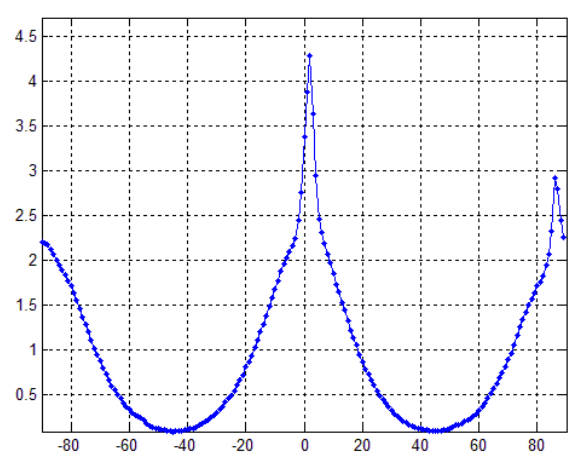

(f)

Fig. 6 (a), (b), (c) show the first 40 lines extracted with the proposed variant from a clockwise rotation of Fig. 1 a by $8^{\circ}$ as well as the integrated arrays of the proposed and $f$-kernel HT variants. Similarly, (d), (e), (f) show the 30 most prominent lines extracted with the proposed variant from a counterclockwise rotation of Fig. $1 \mathrm{~b}$ by $13^{\circ}$ along with the integrated arrays for the rotated and unrotated image.

The proposed method can also be used in applications that require finding prevalent orientations, rather than individual lines. Prevalent orientations correspond to peaks of an "integrated accumulator array", which is formed by adding the contributions of all accumulator array cells that correspond to a certain value of $\theta$. 
For example, the ellipse-based HT has been used with success for finding striped formations in digital images of marble slabs [19]. Here, we seek prevalent orientations in rotated images of noisy buildings in order to rectify the images. Fig. 6a shows the 40 most prominent lines found with the proposed HT for a clockwise rotation of Fig. 1a by $8^{\circ}$ and Figs. $6 \mathrm{~b}$ and $6 \mathrm{c}$ show correct peaks at $-8^{\circ}$ and $82^{\circ}$ of the integrated arrays - normalized with respect to the number of nonzero bins - for the proposed and $f$-kernel variants. The smoother integrated array of Fig. $6 \mathrm{~b}$ is due to the higher spread of votes to neighboring bins of the proposed algorithm. Similarly, Fig. $6 \mathrm{~d}$ shows a rotation of Fig. $1 \mathrm{~b}$ by $13^{\circ}$ counter-clockwise which corresponds to the angular difference of corresponding peaks in the integrated arrays of the rotated and unrotated images shown in Figs. $6 \mathrm{e}$ and $6 \mathrm{f}$.

The next experiment performs image rectification on the perspectively deformed thermal IR image of Fig. 1c. Fig. 7a shows the 16 most prominent lines extracted with the proposed HT variant. Fig. $7 \mathrm{~b}$ shows the corresponding integrated array with one clear peak at $-3^{\circ}$ (nearly parallel lines) and one cluster of converging lines in the neighborhood of $-89^{\circ}$. Using $k$-means to cluster the lines and RANSAC to robustly estimate the two vanishing points we evaluate the 3D projectivity matrix and rectify the image (see for details [29]).

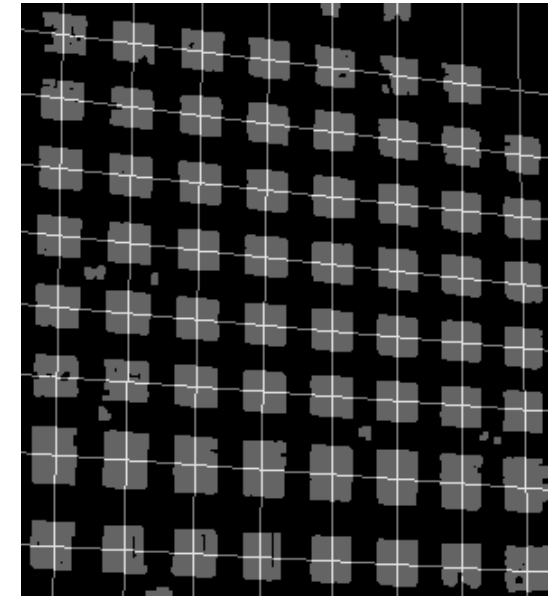

(a)

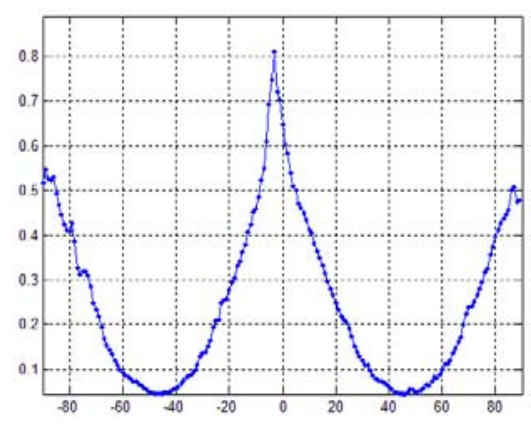

(b)

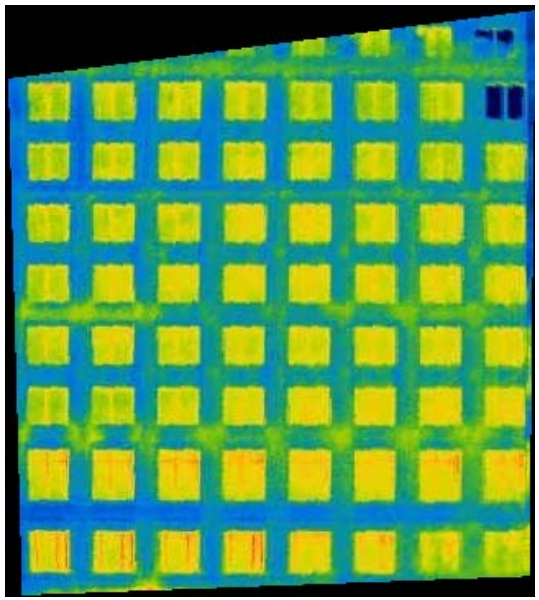

(c)

Fig. 7 (a) 16 lines extracted with the proposed method, b) the integrated array and c) the rectified image.

Finally, the proposed HT method has been applied to a completely different problem, that of skew detection and correction which is typical in scanned document image analysis. Fig. 8a shows a typical skewed document image obtained from a scanning device. To this image we apply the RLSA smearing technique according to which a sequence of white consecutive pixels is converted to black provided that the length of the sequence does not exceed a predetermined threshold. Fig. $8 \mathrm{~b}$ shows the MBRs on the result of RLSA smoothing with a threshold $\mathrm{H}=5$ followed by Otsu's binarization and, inversion and Fig. 8c shows the corresponding integrated array with a clear peak at -3 degrees. Fig. 8d shows the skew corrected document image obtained with a $3^{\circ}$ rotation.

\section{Conclusions}

The proposed region-based technique has been shown to accurately detect lines in noisy night images of buildings. Experimental results and comparisons show the superiority of our method in terms of line localization and detection of linear formations with respect to two other region-based HT variants, namely, a weighted HT method that uses shape descriptors computed from the best fitted ellipses to the regions and a region-based HT variant that casts votes in the accumulator array using the kernel of eq. (14). The method presented in this work can also be used in order to detect and correct skew in scanned documents in document analysis applications. 


\section{Acknowledgment}

This research has been co-funded by the EU (European Social Fund) and Greek national resources under the framework of the "Archimedes III: Funding of Research Groups in TEI of Athens" project of the "Education \& Lifelong Learning" Operational Programme.

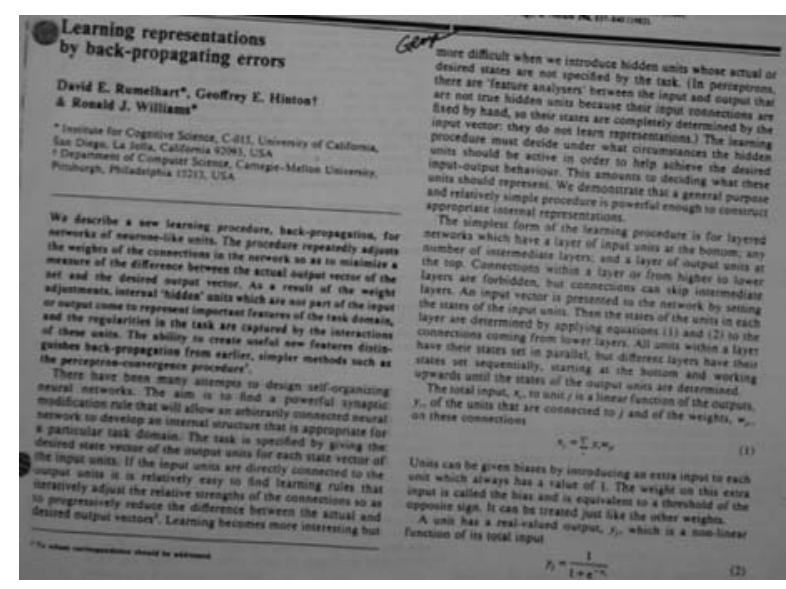

(a)

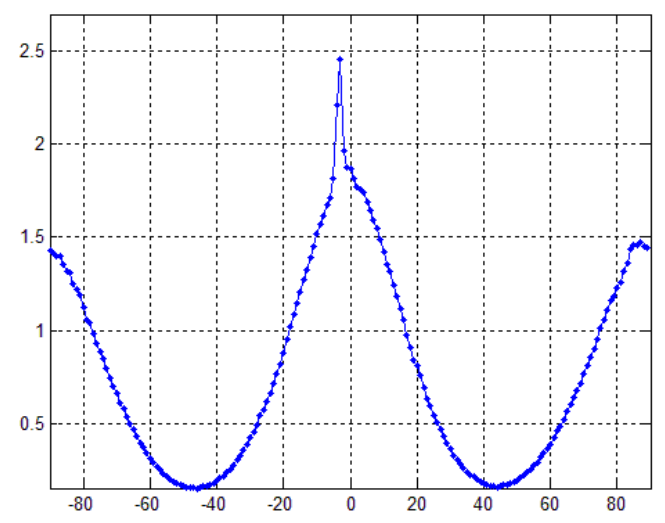

(c)

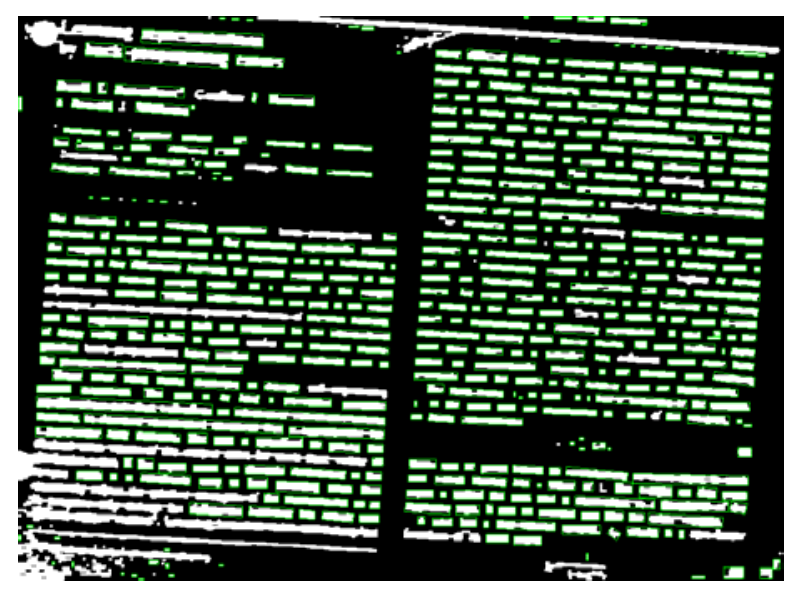

(b)

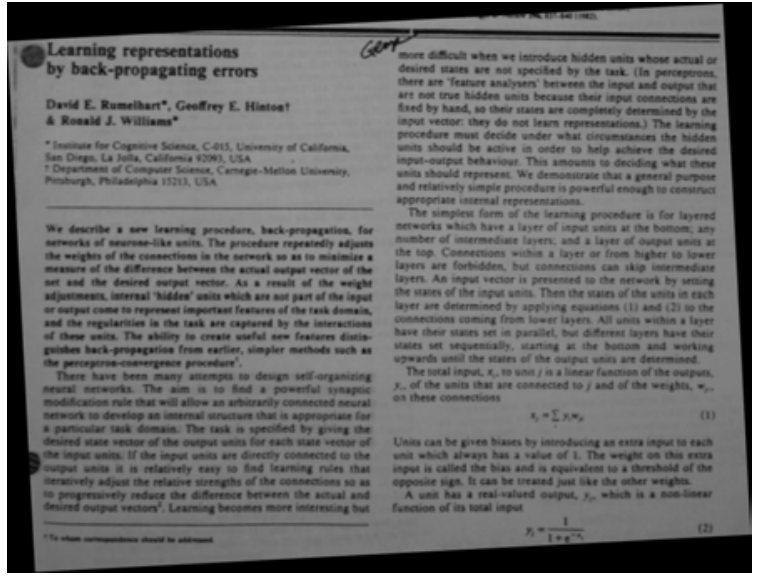

(d)

Fig. 8 (a) The original scanned document, (b) the MBRs on the result of applying RLSA to the binarized and inverted image, (c) the corresponding integrated array, and (d) the skew-corrected document.

\section{References}

[1] M.Y. Yang, W. Forstner, "Feature evaluation for building facade images - an empirical study", Technical Report, Inst. of Geodesy and Geoinformation, Department of Photogrammetry, University of Bonn, 2011.

[2] O. Teboul, L. Simon, P. Koutsourakis, N. Paragios, "Segmentation of building facades using procedural shape priors", Proc. IEEE Conf. on Computer Vision and Pattern Recognition, 3105-3112, 2010.

[3] P. Muller, G. Zeng, P. Wonka, L. VanGool, "Image-based procedural modelling of facades", Proc. ACM SIGGRAPH 07/ACM Transactions on Graphics, 26(3), 2007.

[4] M. Lipp, P. Wonkay, M. Wimmer, "Interactive visual editing of grammars for procedural architecture", ACM Transactions on Graphics, 27(3), 2008.

[5] P. Debevec, C. Taylor, J. Malik, "Modeling and rendering architecture from photographs: a hybrid geometry and image-based approach", Proc. ACM SIGGRAPH 96, 11-20, 1996. 
[6] R. Szeliski, H.-Y. Shum, "Creating full view panoramic image mosaics and environment maps", Proc. ACM SIGGRAPH'97, 251-258, 1997.

[7] M. Brown, D. Lowe, “Automatic panoramic image stitching using invariant features", International Journal of Computer Vision, 74(1): 59-73, 2007.

[8] B. Micusik, J. Kosecka, "Piecewise planar city 3D modeling from street view panoramic sequences", Proc. IEEE Conference on Computer Vision and Pattern Recognition (CVPR), Miami, USA, 2009.

[9] J.A. Lee, Y. Kin-Choong, A.Y. Chia, "Robust matching of building facades under large viewpoint changes", Proc. IEEE 12th International Conference on Computer Vision, Kyoto, Japan, 1258-1264, 2009.

[10] Y. Li, L.G. Shapiro, "Consistent line clusters for building recognition in CBIR", Proc. 16th International Conference on Pattern Recognition (ICPR 02), 3:952- 956, 2002.

[11] X. Yuan, C-T. Li, "CBIR approach to building image retrieval based on invariant characteristics in Hough domain", Proc. IEEE International Conference on Acoustics, Speech and Signal Processing (ICASSP 08), 1209-1212, 2008.

[12] L. Van Gool, G. Zeng, F. Van Den Borre, P. Müller, "Towards mass-produced building models", Photogrammetric Image Analysis, 209-220, 2007.

[13] S.G. Narasimhan, Models and Algorithms for Vision through the Atmosphere, Ph.D. Thesis, Columbia University, 2003.

[14] V.F. Leavers, “Which Hough transform?”, CVGIP: Image Understanding, 58(2):250-264, 1993.

[15] J. Illingworth, J. Kittler, "A survey of the HoughTransform", Computer Vision, Graphics, and Image Processing, 44(1):87-116, 1988.

[16] M. Magee, J. Aggarwal, "Determining vanishing points from perspective images", Computer Vision, Graphics, and Image Processing, 26(2):256-267, 1984.

[17] T. Tuytelaars, L. Gool, M. Proesmans, T. Moons, "The cascaded Hough transform as an aid in aerial image interpretation", Proc. Sixth Int'l Conf. on Computer Vision (ICCV-98), Bombay, India, 67-72, 1998.

[18] D. Liebowitz, A. Zisserman, "Metric rectification for perspective images of planes", Proc. Conference on Computer Vision and Pattern Recognition (CVPR '98), IEEE Computer Society, Santa Barbara, CA, USA, 482-488, 1998.

[19] S. Perantonis, N. Vassilas, Th. Tsenoglou, K. Seretis, "Robust line detection using weighted region based Hough transform", Electronics Letters, 34(7):648-650, 1998.

[20] S. Perantonis, N. Vassilas, K. Seretis, Th. Tsenoglou, "A novel weighted Hough transform for robust detection of linear formations", in Recent advances in information science and technology, ed. N.E. Mastorakis, (World Scientific Publishing), 233-237, 1998.

[21] N. Vassilas, S. Perantonis, E. Charou, Th. Tsenoglou, M. Stefouli, S. Varoufakis, "Delineation of lineaments from satellite data based on efficient neural network and pattern recognition techniques", Proc. Second Hellenic Conference on AI, Thessaloniki, Greece, 355-365, 2002.

[22] N. Otsu, "A threshold selection method from gray-level histograms", IEEE Transactions on Systems, Man and Cybernetics 9(1):62-66, 1979.

[23] P.L. Rosin, "Measuring rectangularity", Machine Vision and Applications, 11(4):191-196, 1999.

[24] M.A. Fischler, R.C. Bolles, "Random sample consensus: a paradigm for model fitting with applications to image analysis and automated cartography", Communications of ACM, 24:381-395, 1981.

[25] http://www.mathworks.com/matlabcentral/fileexchan ge/31126-2d-minimal-bounding-box

[26] K.R. Castleman, Digital Image Processing, Prentice Hall, 1996.

[27] P.L. Palmer, J. Kittler, M. Petrou, “An optimizing line finder using a Hough transform algorithm”, Computer Vision and Image Understanding, 67(1):1-23, 1997.

[28] N. Vassilas, Th. Tsenoglou D. Ghazanfarpour, "Robust region-based line detection from poor quality images of aligned rectangular objects", Proc. IASTED Int. Conf. on Signal Processing, Pattern Recognition and Applications (SPPRA 2012), Crete, Greece, 35-42, 2012.

[29] R.Hartley, A. Zisserman, Multiple View Geometry in Computer Vision, Cambridge University Press, 2000 . 